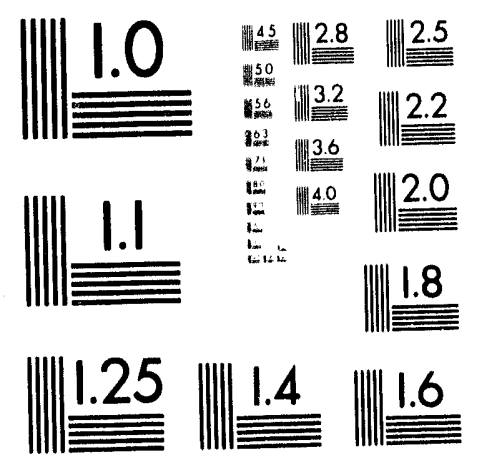



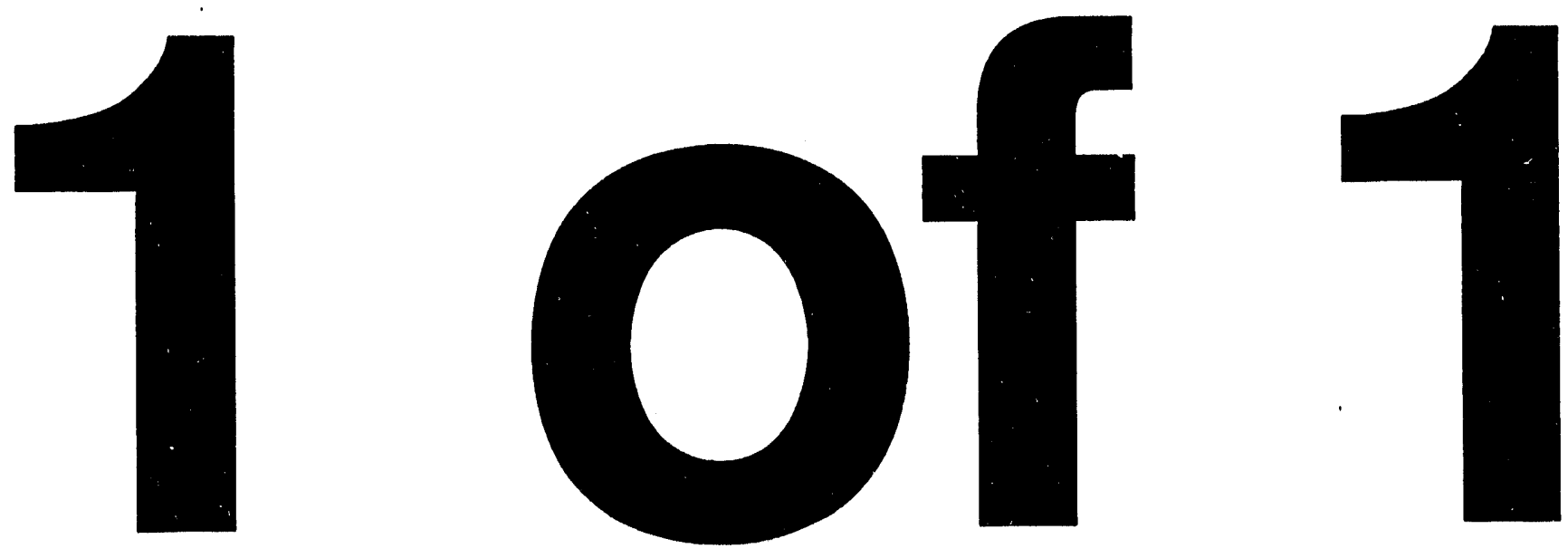


$$
\text { (0.7.930709-2) }
$$

UCRL- JC-112\%93

PREPRINT

\title{
IMAP: A Complete Ion Micro-Analysis Package for the Nuclear Microprobe
}

\author{
A.J. Antolak, G.S. Bench, \\ M.L. Hildner, and D.H. Morse
}

This paper was prepared for submittal to the 11th International Conference on Ion Beam Analysis

Balatonfüred, Hungary

July 5-10, 1993

June 7, 1993

This is a preprint of a paper intended for publication in a journal or proceedings. Since changes may be made before publication, this preprint is made available with the understanding that it will not be cited or reproduced without the permission of the author.

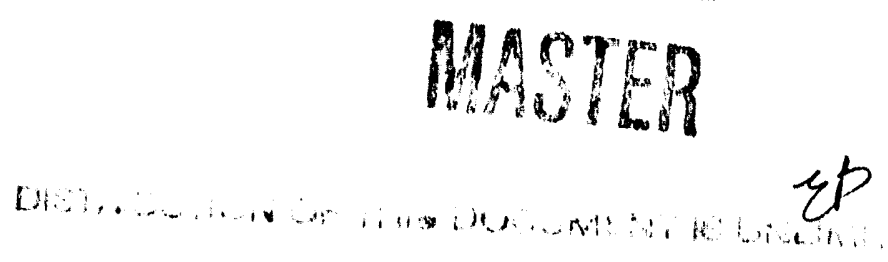




\section{DISCLAIMER}

This document was prepared as an account of work sponsored by an agency of the United States Govermment. Neither the United States Government nor the Uni versity of California nor any of their employees, makes any warranty, express or implied, or assumes any legal liability or responsibility for the accuracy, completeness, or usefulness of any information, apparatus, product, or process disclosed, or represents that its use would not infringe privately owned rights. Reference herein to any specific commercial products, process, or service by trade name, trademark, manufacturer, or otherwise, does not necessarily constitute or imply its endorsement, recommendation, or favoring by the United States Government or the University of California. The views and opinions of authors expressed herein do not necessarily state or reflect those of the United States Government or the University of Califormiz, and shall not be used for advertising or product endorsement purposes. 


\title{
IMAP: A Complete Ion Micro-Analysis Package for the Nuclear Microprobe*
}

\author{
A. J. Antolak ${ }^{\S}$, G. S. Bench ${ }^{\dagger}$, M. L. Hildner ${ }^{\S}$, and D. H. Morse ${ }^{\S}$ \\ §Sandia National Laboratories \\ Livermore, CA 94551 \\ ${ }^{\dagger}$ Lawrence Livermore National Laboratory \\ Livermore, CA 94550
}

\begin{abstract}
Microprobe techniques using scanned, focused $\mathrm{MeV}$ ions are routinely used in Livermore for materials characterization. Comprehensive data analysis with these techniques is accomplished with the computer software package IMAP, for Ion Micro-Analysis Package. IMAP consists of a set of command language procedures for data processing and quantitative spectral analysis. Deconvolution of the data is achieved by spawning sub-processes within IMAP which execute analysis codes for each specific microprobe technique. IMAP is structured to rapidly analyze individual spectra or multi-dimensional data blocks which classify individual events by the two scanning dimensions, the energy of the detected radiation and, when necessary, one sample rotation dimension. Several examples are presented to demonstrate the utility of the package.
\end{abstract}

*This work was supported by the U.S. Depurtment of Energy under SNL Contract No. DE-AC04-76P00789 and LLNL Contract No. W-7405-ENG-48. 


\section{Introduction}

The need for quantitative, high resolution characterization of materials has led to the development of microprobes using focused $\mathrm{MeV}$ ion beams for particle-induced $\mathrm{x}$-ray emission (PIXE), ion backscattering spectroscopy (IBS), ion microtomography (IMT), particle-induced gamma emission (PIGE), scanning transmission ion microscopy (STIM) and ion microtomography (IMT). For the first three of these techniques, the ion beam can be used in a stationary spot mode for the collection of individual spectra from a particular specimen region or it can be scanned across the sample. STIM, stereoscopic imaging techniques and IMT require the sample to be scanned and, in the latter two cases, rotated. The utilization of a scanned beam allows data collection in the form of multidimensional data blocks which usually consist of the two beam scanning dimensions, the energy from the detected radiation and, when necessary, one sample rotation dimension. It is often necessary to extract spectral and/or positional information from sub-regions within these data blocks for detailed analysis. Further, a means to unfold and analyze the spectral data is necessary for quantitative materials analysis.

This paper describes the data analysis computer package IMAP (Ion MicroAnalysis Package) developed for IMAG (Ion Micro-Analysis Group) in Livermore. IMAP is a collection of procedures built upon PV-WAVETM software [1] and currently running on SUN'M computer workstations. IMAP is easily transportable between different computer systems and because it uses high-level, interpretive commands and procedures, it allows simplified data access, reduction, and analyses. In IMAP, large multi-dimensional dácasets, image files or individual spectra can be processed rapidly and accurately. Spectral and/or spatial information can be readily extracted from multidimensional data sets for further study. By spawning child processes, IMAP can link to existing software for quantitative PIXE, IBS, IMT, and PIGE analysis of the relevant data. These features provide the experimentalist with the tools needed to extract information essential for accurate materials characterization.

\section{Structure of IMAP}

IMAP presently consists of approximately 100 coupled command language procedure and function subroutines. The sequence of data processing steps is controlled by the user through either menu-, mouse- or keyboard-driven input. IMAP is designed to perform powerful data analysis in a user-friendly environment in order to retain its tractability for the experimentalist. For example, self-consistency checks are utilized 
extensively throughout the package to avoid program crashes and calculation errors. The modular structure allows extensions or modifications to be incorporated in a simple and logical manner. In nearly all stages of an analysis session, the user can select from various options to further manipulate a spectrum or image, save or print the current results, or quit and move on to other data. These and other features are built into the package making it a versatile tool for nuclear microprobe data processing.

A flowchart of the IMAP package is shown in Figure 1. Window management, plotting, and image display are driven by the visualisation software thereby relieving the need to develop specialized machine-dependent codes for these functions. After completion of a particular processing step, control is returned to a main menu and the user queried for further action. Upon entering IMAP, the user is prompted for the type of analysis to be conducted, i.e., PIXE, IBS, IMT, etc. Control is then directed to the appropriate block of procedures dealing with the specified technique.

Our data acquisition procedure is similar to that of Total Quantitative Scanning Analysis (TQSA) [2]. All data from a particular detector are recorded during an ion beam irradiation and can be stored either as a spectrum or a multi-dimensional data block for analysis with IMAP. For PIXE, STIM, IBS or PIGE the data block is a cube consisting of the two scanning dimensions and the energy. Spatial maps can be readily generated from the cube by using a workstation mouse to set windows around certain energy ranges of the total accumulated energy spectrum. Using these maps the energy spectrum from any shaped feature of interest can be extracted for quantitative analysis. A particular feature is defined on a map by using the mouse to trace out its shape with a series of connecting straight line segments. In a sequence of interactive steps, the analyst can move from spectral displays to spatial maps and back to spectra for quantitative analysis of a given feature. As all data are stored during data collection, no data are lost, so elements not visible in the total spectrum, but present in some localized concentration, may be enhanced when the spectrum from that region is inspected. Quantitative spectrum analysis results can be calculated immediately and stored or printed; or, the spectrum itself can be stored with other spectra and spatial maps in a subdirectory of the user's local workspace for subsequent analysis. Ratios of corresponding pixel values from two spatial maps can be computed to highlight differences in specimen composition. Line scans of intensity versus position can also be generated for any given energy interval in the total accumulated energy spectrum. The user has the option to display spatial maps as greyscale or color images, contour or surface plots and combinations thereof. In addition, the spatial map data can be reallocated into a smaller cube, smoothed with the choice of several different filters or printed in hard copy format. Hard copy output can also be 
obtained from previously stored analysed data. STIM data can be displayed as maps depicting the mean or median energy loss, or moments about the mean energy loss. Subregions within these residual ion energy maps can be examined for more detailed and quantitative analysis.

The versatility of IMAP in extracting quantitative data from a three-dimensional data set is illustrated in figure 2 . The sample is a $0.5 \mathrm{~mm}$ diameter direct drive inertial confinement fusion sphere which was examined via PIXE. Figure 2a shows the bromine $\mathrm{K} \alpha \mathrm{x}$-ray distribution within the $800 \times 800 \mu \mathrm{m}^{2}$ scan area. The data were acquired with a $3 \mathrm{MeV}$ proton beam focused to a $25 \mu \mathrm{m}$ diameter spot and the image consists of $32 \times 32$ pixels. Bromine is used as a diagnostic trace element for fusion performance tests and was verified to be uniformly distributed at a concentration of $3 \mathrm{wt} \%$ throughout the outer layer of the sphere by this analysis. The $\mathrm{x}$-ray energy spectrum from the sphere was extracted from the cube using the $\mathrm{Br} \mathrm{K} \alpha$ distribution to define the boundaries of the sphere shown in figure $2 \mathrm{~b}$. Identifiable elements in this spectrum include $\mathrm{Br}, \mathrm{I}, \mathrm{Fe}$ and possibly $\mathrm{Cu}$. Figure $2 \mathrm{c}$ shows the detected $\mathrm{x}$-ray intensity distribution over the range 2.54 to $2.70 \mathrm{keV}$ (chlorine $x$ rays fall within this energy window). Data are only detected from the right half of the image of the sphere in this map as low energy $x$ rays produced from the side further from the detector are heavily attenuated by the sample. Figure $2 \mathrm{c}$ reveals a high localization of $\mathrm{x}$-rays (black pixel) towards the bottom left of the sphere. An x-ray energy spectrum extracted from this region is displayed in figure $2 \mathrm{~d}$. In addition to $\mathrm{Br}$ and $\mathrm{I}$ this spectrum reveals $\mathrm{K}$ and $\mathrm{Cl}$ peaks that were not previously visible. Quantitative analysis revealed that the black pixel in figure $2 \mathrm{c}$ was most likely due to a $\mathrm{KCl}$ contaminant on the surface of the sphere.

For each analysis technique, processing of like spectra for quantitative analysis is available in batch mode. Look up tables can be used to calibrate energy spectra, to identify the most likely element associated with a user-specified energy and to label particular spectrum features if desired. We have developed our own software analysis package PIXE-Fit (PIXEF) [3] for analyzing particle-induced x-ray emission data. Selfabsorption and secondary fluorescence effects are treated explicitly for $\mathrm{K}$ and $\mathrm{L}$ shell $\mathrm{x}$ rays and data bases for $\mathrm{x}$-ray mass attenuation coefficients, photoionization and proton ionization cross-sections, Coster-Kronig transition probabilities, fluorescence yields and relative line intensities have been created or improved, where necessary, from reliable current literature values or by direct measurement. The spectrum fitting code utilizes nonlinear least squares fitting techniques $[4,5]$ once the background has been subtracted from the spectrum. The background is estimated using peak filing techniques. Figure 3 shows a $3-\mathrm{MeV}$ proton spectrum obtained from a $\mathrm{Zn}-\mathrm{Ni}$ standard whose concentration by 
mass was specified as $29.9 \% \mathrm{Ni}$. The dotted line corresponds to the raw data, the dashed line is the estimated background, whilst the solid line represents the fit to the peaks in the raw data. PIXEF yields a Ni concentration of $30.3 \pm 1.7 \mathrm{wt} \%$. If secondary fluorescence effects were not included in the spectrum analysis, the nickel concentration would be calculated to be $38.1 \pm 2.3 \mathrm{wt} \%$.

For IBS data we have adapted the program RUMP [6] to produce spectral output accessible to IMAP's format for input and also have incorporated the program TRIM [7] for ion transport calculations. Tentative plans include incorporating the GISA program for backscattering analysis [8] since it treats non-Rutherford scattering cross-sections, electronic screening, improved straggling models and nonlinear detector response. Likewise, IMAP has routines to extract quantitative information from PIGE or STIM spectra.

For IMT, data can be displayed as either reconstructed images, sinograms or projections. Measured residual ion energies are read in as a four-dimensional data block and subprocesses are spawned to convert them to projected densities The raw data can be further processed to remove spurious events. Reconstructed images are obtained by spawning a suite of LLNL-developed reconstruction algorithms [9]. Limited angle data can also be processed and full three-dimensional rendering of the specimen is possible. Subregions of any displayed image can be extracted for more detailed and quantitative analysis and hard copy output can be produced at any stage of the analysis. Figure 4 shows IMT data of a production reactor fuel pellet analysed using IMAP. The target consists of a lithium aluminate core surrounded by porous carbon which allows for tritium retention. The outer coatings consist of pyrolytic carbon and $\mathrm{SiC}$ layers formed by chemical vapour deposition. These layers function as either chemical or structural support for retaining the core material and its products. IMT data were acquired using a $12 \mathrm{MeV}$ proton beam focused to a spot of $5 \mu \mathrm{m}$. Thirty contiguous slices were acquired from the top one third of the pellet. Each slice was separated by $5 \mu \mathrm{m}$ and 189 evenly spaced projections were recorded over $360^{\circ}$. Figure 4 shows the sinogram and reconstructed image of the $25^{\text {th }}$ slice. The individual coatings are clearly discernible and there appears to be a low density region near the kernel which may correspond to a delaminated layer. The color bar calibrates the electron density in the reconstructed image. Below the color bar is a density lineout which corresponds to the black trace shown in the reconstructed image. Variations in density arising from each layer are visible as is the significant drop in density across the internal flaw. 


\section{Conclusion}

IMAP is a compact, easily transportable and versatile MeV nuclear microbeam software analysis package built on a PV-WAVETM platform for data visualisation. We currently use IMAP to analyse all PIXE, IBS, STIM, IMT and PIGE data and it has satisfied our expectations. Self consistency checks are used extensively throughout the package and it is difficult to create program crashes or calculation errors. Because it uses both highlevel, interpretive commands and is capable of utilizing external analysis codes, it allows simplified data access, reduction and analysis. These features provide the experimentalist with an effective tool for quantitative materials characterization.

\section{Acknowledgments}

The authors wish to thank Dale Heikkinen, Alex Friz and Art Pontau for providing helpful input, suggestions and comments regarding numerous aspects of the package. Dale and Art also participated in acquiring data for the examples used in this paper.

\section{References}

[1] Precision Visuals Inc, 6230 Lookout Road, Boulder, Colorado 80301, U.S.A.

[2] G. J. F. Legge and I. Hammond, J. Microsc. 117 (1979) 201.

[3] A.J. Antolak, G.S. Bench, and A.M. Friz, in preparation.

[4] E. Clayton PIXAN: The Lucas Heights PIXE analysis computer package, AAEC/M113 (Lucas Heights research laboratories, Menai, Australia, 1986).

[5] P.R. Bevington, Data Reduction and Error Analysis for the Physical Sciences, McGraw-Hill, New York, 1969.

[6] L. Doolittle, Nucl. Instr. and Meth. B9 (1985) 344; B 15 (1986) 227.

[7] J.P. Biersack and L.G. Haggmark, Nucl. Instr. and Meth. 174 (1980) 257.

[8] J. Saarilahti and E. Rauhala, Nucl Instr. and. Meth. B64 (1992) 734.

[9] H.E. Martz, S.G. Azevedo, J.M. Brase, K.E. Waltjen, and D.J. Schneberk, Lawrence Livermore National Laboratory Report UCRL 98492; Int. J. Rad. Appl. and Instr. A41, (1991) 943. 


\section{Figure Captions}

Figure 1. Flow chart depicting the structure of the IMAP package. The term "cube" refers to a multi-dimensional data block.

Figure 2. The bromine $\mathrm{K} \alpha \mathrm{x}$-ray distribution within a $0.8 \times 0.8 \mathrm{~mm}^{2}$ scan area including a direct drive inertial confinement fusion (ICF) sphere is shown in $2 a$. In $2 b$ is the $x$-ray spectrum of only the ICF sphere which was extracted from the acquired PIXE cube data. All identifiable $x$-ray peaks are labelled. The peak at $14.5 \mathrm{keV}$ is from a pulser. The $\mathrm{x}$-ray distribution from the energy range 2.54 to $2.70 \mathrm{keV}$ is shown in $2 \mathrm{c}$. The spectrum corresponding to the black pixel of $2 \mathrm{c}$ is displayed in $2 \mathrm{~d}$. Note the prominant potassium and chlorine peaks.

Figure 3. A 3-MeV proton PIXE spectrum and associated PIXEF fits from a $\mathrm{Zn}-\mathrm{Ni}$ standard. The dotted line is the raw data, the dashed line the background and the solid line represents the fit to the raw data. All identifiable $x$-ray peaks are labelled.

Figure 4. IMT data of a production reactor fuel pellet. The upper left image is a sinogram of the $25^{\text {th }}$ slice of the projection data from the pellet. The upper right image shows the reconstructed data. The image size is $0.6 \times 0.6 \mathrm{~mm}^{2}$. The color bar provides a calibration for the electron densities shown in the reconstructed image. The bottom graph is a line out of the pellet's density across the target including an internal flaw near the inner kernel. The position of the lineout is shown as the black trace in the reconstruction. 


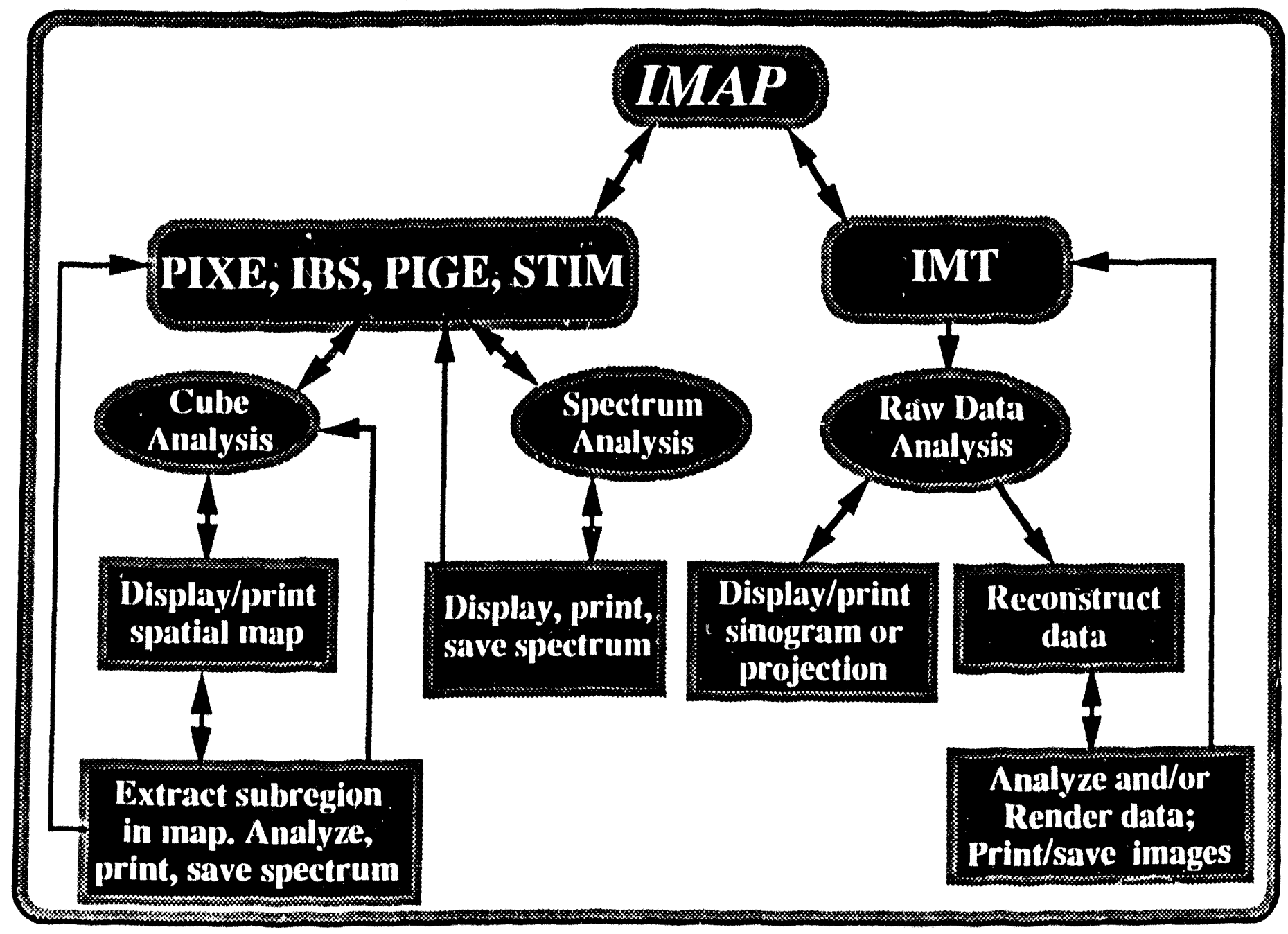




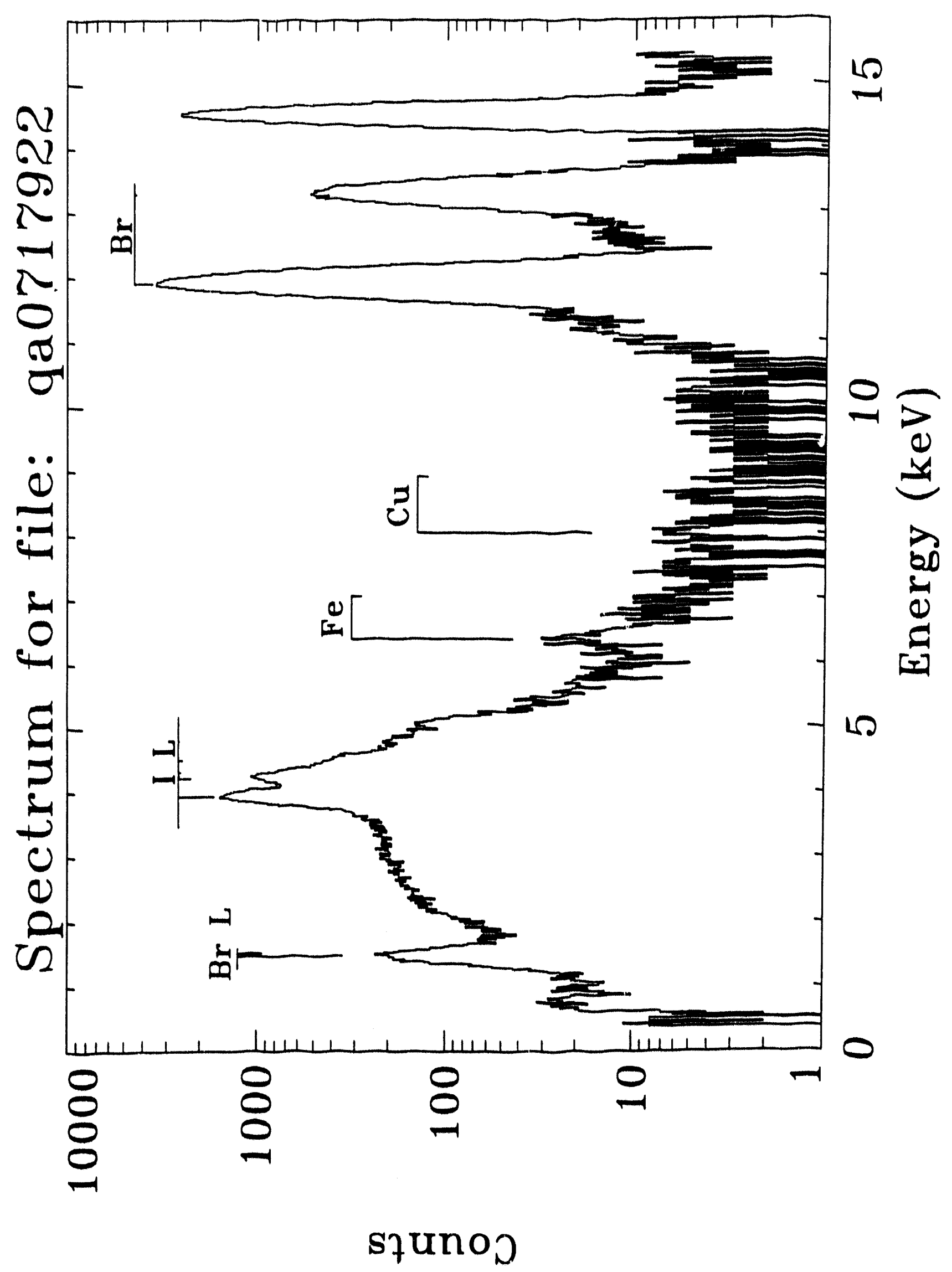




\section{Br k-alpha energy window (11.60 to $12.23 \mathrm{keV}$ )}
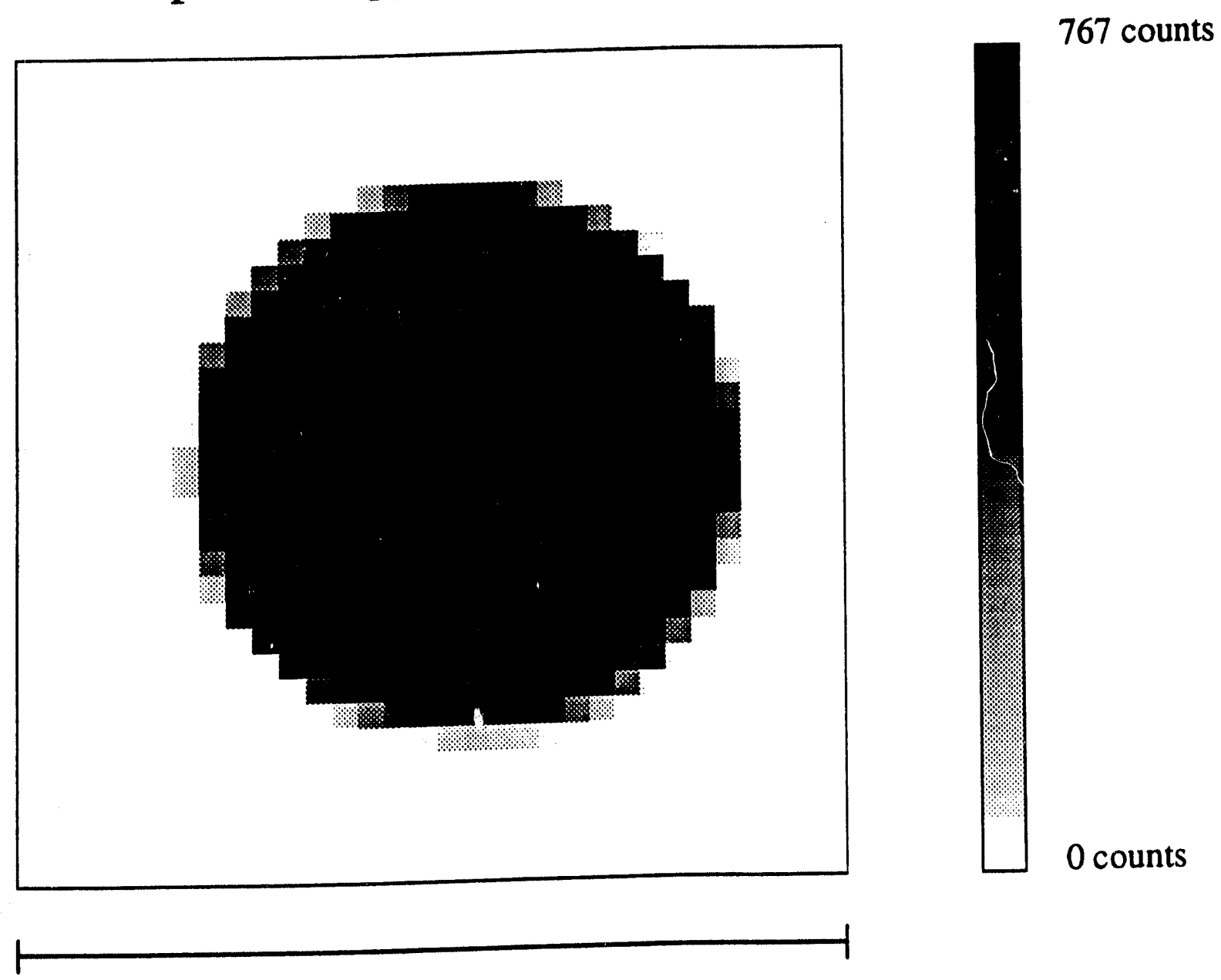
Chlorine energy window (2.54 to $2.70 \mathrm{keV}$ )

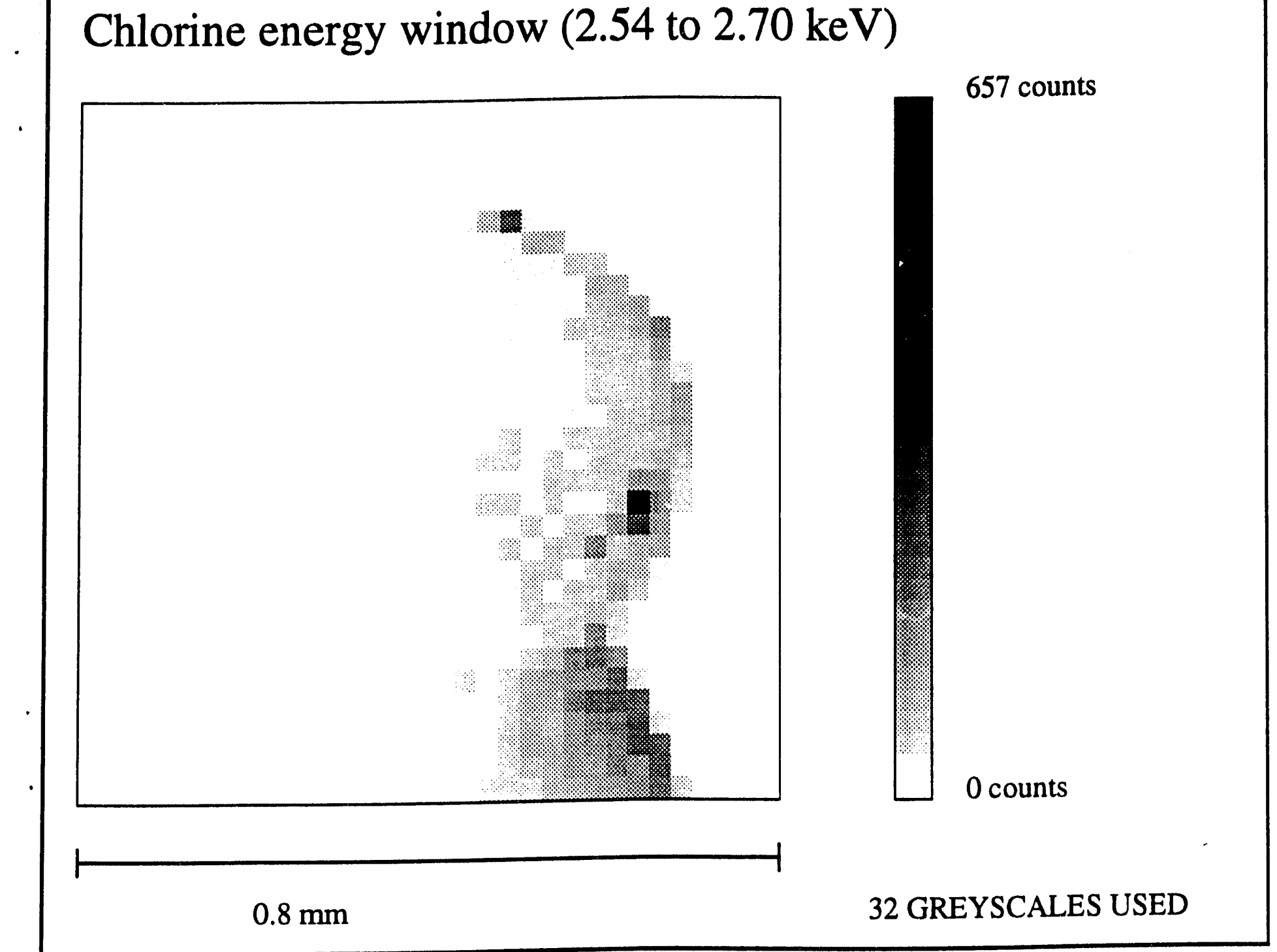




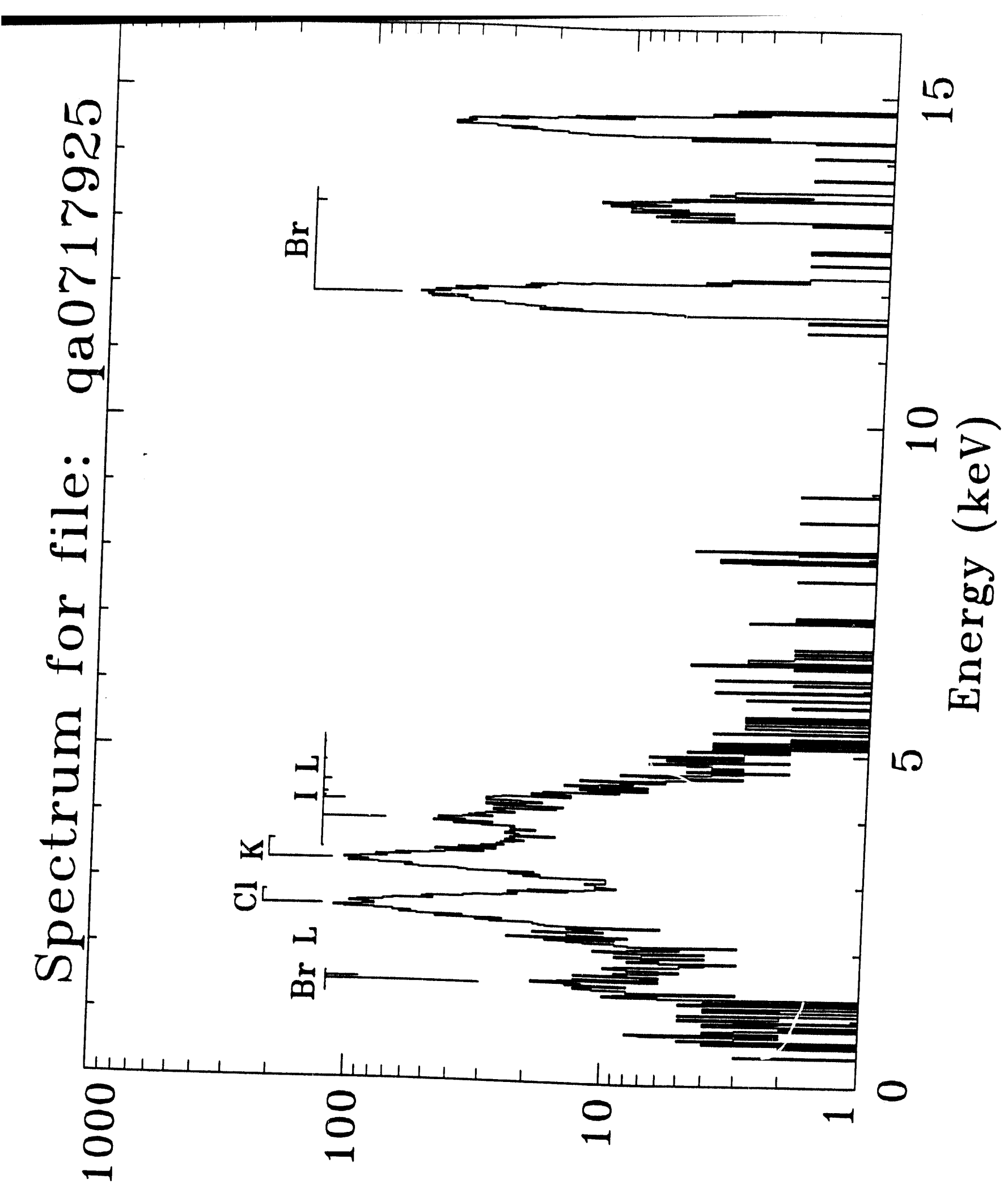

squno: 


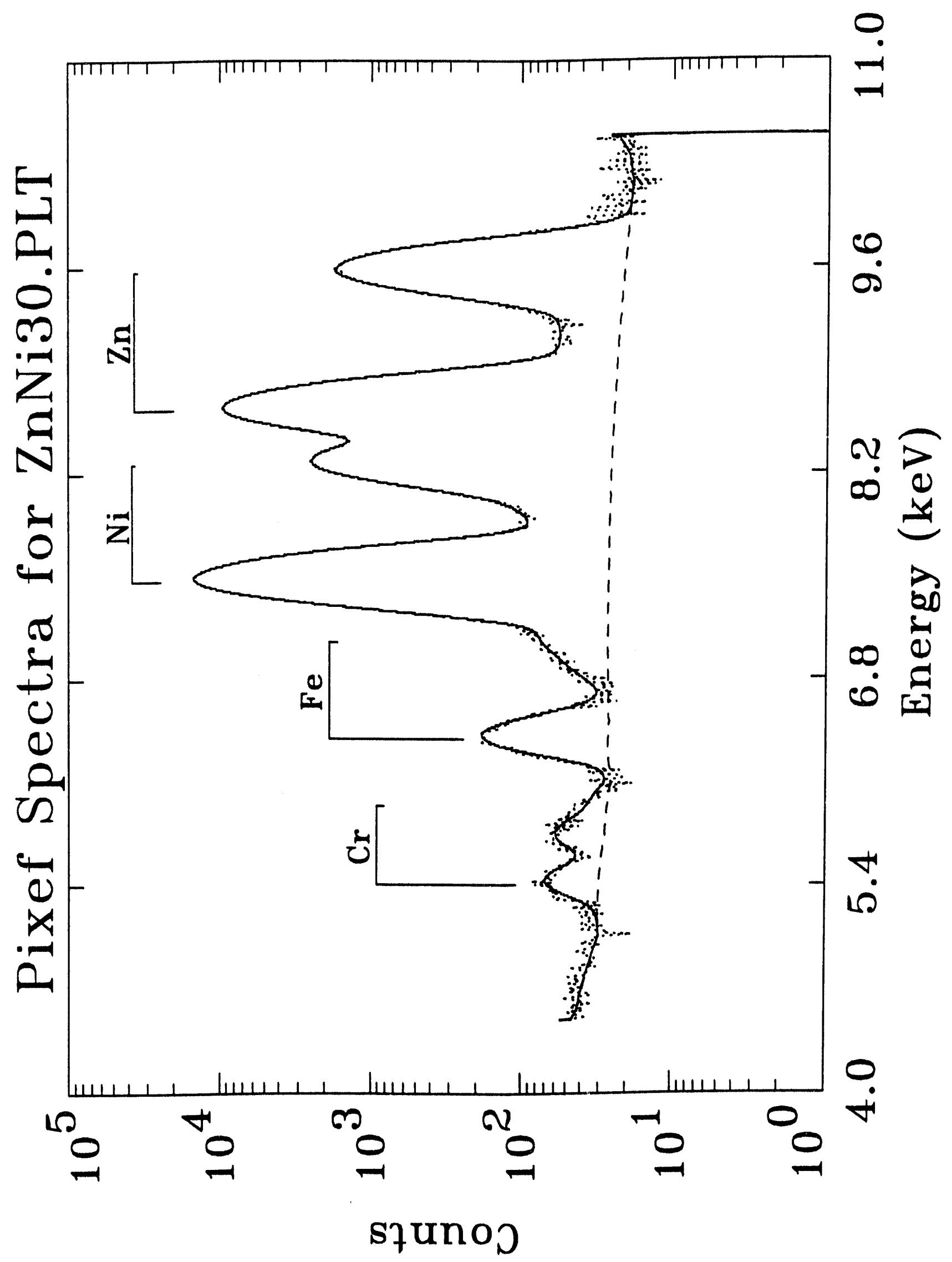




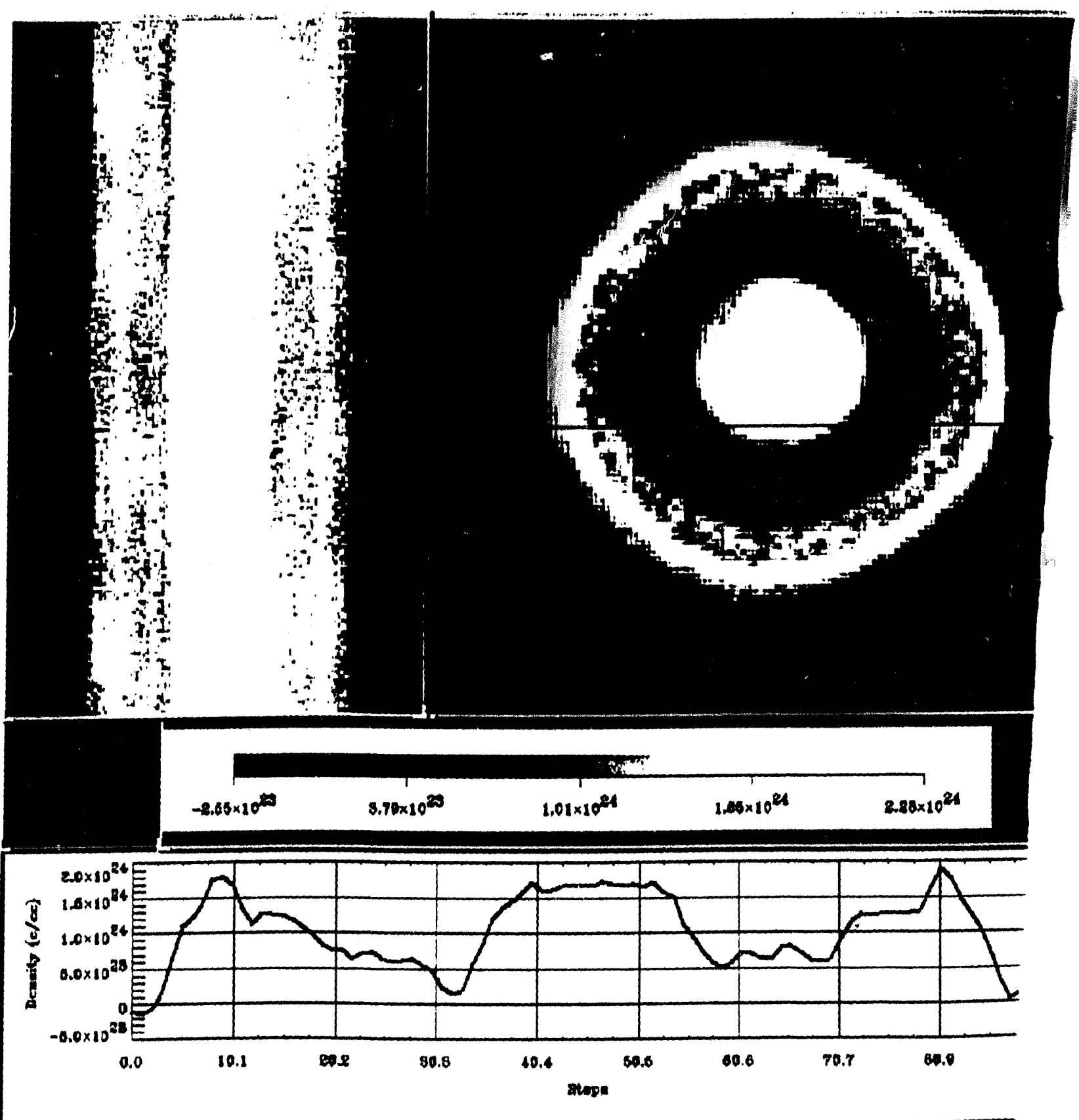




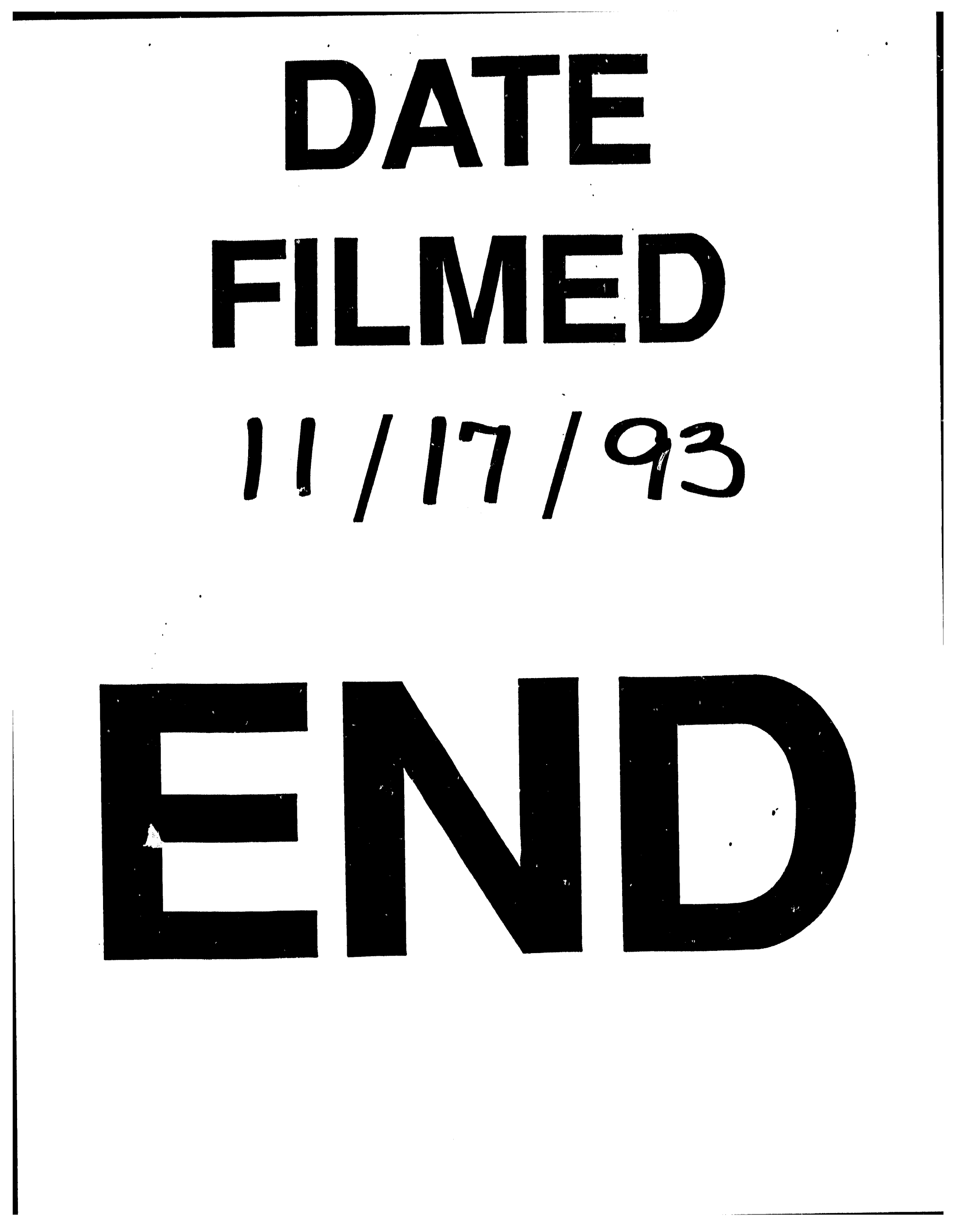




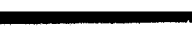

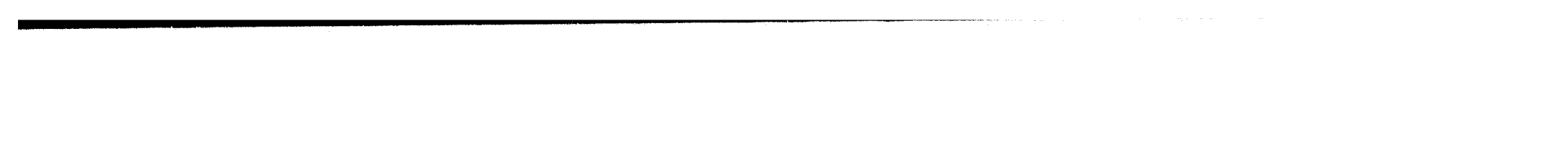

